\title{
Electromyographic Analysis of Thigh Muscles in PNF Patterns of the Lower Extremity: Muscle Activities in the Lengthened Range
}

\author{
Kan HAZAKI $^{1)}$, Noriaki IchiHashi ${ }^{1)}$, ToshiHIRo Morinaga ${ }^{1)}$ \\ 1) Division of Physical Therapy, College of Medical Technology, Kyoto University, \\ 53 Kawahara-cho, Shogoin, Sakyo-ku, Kyoto 606-01, Japan. TEL +81-75-751-3964.
}

J. Phys. Ther. Sci.

8: 29-32, 1996

\begin{abstract}
The purpose of this study was to characterize the activity levels of the thigh muscles (Vastus medialis oblique muscle, VMO; Rectus Femoris muscle, RF; Vastus Lateralis muscle, VL; and Biceps Femoris muscle, BF) during 4 basic patterns of Proprioceptive Neuromuscular Facilitation (PNF) techniques of the lower extremity with the knee straight. Ten healthy adult volunteers $(5$ men and 5 women, with a mean age of $21.7 \pm 3.2$ years) who were knowledgable about PNF patterns were involved in this study. Surface electromyography (Furusawa Labo) was performed and recorded by bipolar miniature silver-silver chloride electrodes, from which rectified filtered electromyograms (RFEMG) obtained. Each PNF pattern was examined under maximal isometric contraction against manual resistance in the lengthened range. Normalized data (\%RFEMG) were analyzed using one-way analysis of variance and Tukey's multiple comparison. The results allowed us to divide PNF patterns into two types: the type containing a flexor component, and that containing an extensor component. In the first type of PNF, \%RFEMG was highest in RF > VL > VMO > BF and in second type of PNF, \%RFEMG was highest in $\mathrm{BF}>\mathrm{VMO}>\mathrm{VL}>\mathrm{RF}$. RF was found to be primarily active in the first type of PNF, while BF was primarily involved in the second type of PNF. VMO tended to be involved in PNF patterns containing components of adduction and external rotation. VL activity did not differ significantly among PNF patterns.
\end{abstract}

Key words: Proprioceptive Neuromuscular Facilitation(PNF), Electromyography, Thigh muscles

(This article was submitted Mar. 4, 1996, and was accepted May 10, 1996)

\section{INTRODUCTION}

Various methods including the manual method and methods using special machines are utilized for muscle strengthening exercises. Among these methods, manual muscle strengthening exercises have several advantages such as easy selection of the patterns of muscle contraction, according to the purpose of the exercise, as well as adaptability of the exercise program to the muscle strength and fatigue of the patient. Among these manual exercises, the PNF technique is relatively well established.
PNF was systematized in the 1940's by Kabat, Knott et al. It has often been used clinically as a manual treatment technique. PNF is characterized by three-dimensional mass movements. Spiral and diagonal movements are adopted in the technique ${ }^{1)}$. These movements are consistent with the skeletal system of bones and joints, and the ligamentous structures. Furthermore, these movements are closely related to activities in daily life ${ }^{1)}$. Because of these consistencies and similarities, the PNF technique has recently been applied to the treatment of sports injuries as well as to improving performance, and promising results have been obtained ${ }^{2)}$. Neurophysiological analysis of PNF as a 
facilitation technique ${ }^{3-7)}$, and usefulness of PNF treatment ${ }^{8-10)}$ have already been reported by numerous investigators, however, there have been few reports of actual muscle activity in PNF.

The purpose of this study was to measure the activity levels of the thigh muscles in four PNF patterns of the unilateral lower extremity and to characterize muscle activity in each PNF pattern.

\section{METHOD}

\section{Subjects}

Ten physical therapy students $(5$ men and 5 women, with a mean age of $21.7 \pm 3.2$ years) with a knowledge of PNF patterns, volunteered for the study. None of the subjects had a history of neurological or orthopedic disorders of the lower extremities or back. All subjects were informed of the objectives of this study and gave consent prior to participating.

\section{Examined muscles}

Four muscles were examined: the vastus medialis oblique muscle (VMO), rectus femoris muscle (RF), vastus lateralis muscle (VL), and biceps femoris muscle $(\mathrm{BF})$ in the right lower extremity.

\section{Examined PNF patterns}

The PNF patterns examined were four unilateral lower extremity patterns: 1) hip flexion, adduction and external rotation (D1F); 2) hip extension, abduction and internal rotation (D1E); 3) hip flexion, abduction and internal rotation (D2F); and 4) hip extension, adduction and external rotation (D2E). Each pattern was tested with the knee straight.

The subjects were positioned supine on a bed. They were wound up adequately and then performed quick stretching, to induce maximum isometric contraction against manual resistance in the lengthened range for 3 seconds.

Each subject performed the exercises three times before the examination to understand the patterns of motion. During measurement, we confirmed that the direction of the movement was diagonal and that all three movement factors were included in the movements. The order of the examined PNF patterns were performed at random in all subjects, and a two-minute rest period was allotted after each pattern to avoid fatigue. The same therapist performed all procedures.

\section{Apparatus}

Each muscular activity was recorded by a surface electromyograph (Furusawa Labo).

Miniature silver-silver chloride electrodes, measuring $8 \mathrm{~mm}$ in diameter were attached to the subject's skin in line with the muscle fibers. A constant distance of $20 \mathrm{~mm}$ was maintained between the centers of electrodes. By bipolar recording using these electrodes, rectified filtered electromyograms (RFEMG) were obtained. Impedances below $10 \mathrm{Kohm}$ were accepted. A common ground electrode was positioned over the right external ear. Stability of myograms was also checked.

\section{Statistical analysis}

From the RFEMG obtained, we calculated the mean RFEMG value of each muscle for 3 seconds in each PNF pattern. The data of the quadriceps muscles were normalized by the mean RFEMG value during maximal isometric contraction of each muscle with the knee extended at 0 degree for 3 seconds. The data of biceps femoris muscle were also normalized by the mean RFEMG value with the knee flexed at 0 degree for 3 seconds. This was done to correct for the differing absolute levels of RFEMG in different subjects. \%RFEMG was calculated as follows:

$\%$ RFEMG $=$

$\{$ (the mean RFEMG value of each muscle in PNF patterns) $\div$ (the mean RFEMG value of each muscle in the maximal isometric contraction) $\} \times 100$.

The mean of \%RFEMG of each muscle was then analyzed using two-way analysis of variance and Tukey's multiple comparison for each pattern.

\section{RESULTS}

Means and standard deviations of the \%RFEMG value for the $\mathrm{VMO}, \mathrm{RF}, \mathrm{VL}$ and $\mathrm{BF}$ in the four lower extremity PNF patterns are shown in the Table 1. In PNF patterns that contained the flexor component (D1F, D2F), \%RFEMG was highest in $\mathrm{RF}>\mathrm{VL}>\mathrm{VMO}>\mathrm{BF}$. In PNF patterns that contained the extensor component (D1E, D2E), \%RFEMG was highest in the opposite order (BF > 
Table 1. Means and standard deviation of the \%RFEMG value for each muscle in four PNF patterns $(\mathrm{N}=10)$

\begin{tabular}{|c|c|c|c|c|c|c|c|c|}
\hline \multirow[b]{2}{*}{ Muscle } & \multicolumn{2}{|l|}{ D1F } & \multicolumn{2}{|l|}{ D1E } & \multicolumn{2}{|l|}{$\mathrm{D} 2 \mathrm{~F}$} & \multicolumn{2}{|c|}{ D2E } \\
\hline & $X$ & SD & $X$ & SD & $X$ & SD & $X$ & SD \\
\hline VMO & 42.5 & 34.3 & 25.0 & 13.5 & 12.8 & 10.3 & 37.7 & 36.3 \\
\hline $\mathrm{RF}$ & 85.1 & 48.0 & 12.0 & 15.6 & 81.5 & 39.8 & 15.0 & 19.0 \\
\hline VL & 50.7 & 28.3 & 19.5 & 21.7 & 21.9 & 18.5 & 31.0 & 42.6 \\
\hline $\mathrm{BF}$ & 22.3 & 11.1 & 136.2 & 73.7 & 10.8 & 9.0 & 96.0 & 86.4 \\
\hline
\end{tabular}

$\mathrm{VMO}>\mathrm{VL}>\mathrm{RF}$ ). Therefore, the activity of each muscle could be divided into two components (flexor and extensor). The flexor component was remarkably higher in the RF, while the extensor component was remarkably higher in the BF.

The \%RFEMG of VMO did not differ significantly among the different PNF patterns, although it was slightly higher in the patterns containing components of adduction and external rotation (D1F, D2E). The \%RFEMG of RF in the patterns containing flexor components was significantly higher than that in patterns containing extensor components $(p<0.05)$. Regarding the \%RFEMG of the VL in the various patterns, the D1F pattern showed the highest activity of the four patterns, but the PNF patterns did not significantly influence this parameter. In contrast to the finding for RF, the \% RFEMG of BF in patterns containing an extensor component was significantly higher than in patterns containing a flexor component $(\mathrm{p}<0.05)$.

\section{DISCUSSION}

Although the PNF technique has been widely used as an effective manual muscle strengthening and facilitation method, there have been few studies that recorded muscular activities during PNF11-13). Voss et al. systematized the PNF technique based on the alignment characteristics of the individual muscles, palpation of muscle action in normal and pathological subjects, and studies on the maximal extension of each muscle1). They did not, however, actually analyze muscle activities, therefore, their theory contains many empirical factors. For this reason, we investigated the activity of the thigh muscles of the lower extremity (VMO, $\mathrm{RF}, \mathrm{VL}$ and $\mathrm{BF}$ ) in four PNF patterns.

The PNF patterns could be divided into two components: flexion patterns and extension patterns. Of the two joints muscles, RF showed high muscle activities in the flexion patterns, whereas
BF showed high muscle activities in the extension patterns. There were no significant difference between flexion and extension patterns in the one joint muscles (VMO and VL). VMO was more active during patterns with adduction and external rotation components.

Voss et al. described the selection of patterns for different weakened musclesi). They reported that the patterns including flexion components $(\mathrm{D} 1 \mathrm{~F}$, D2F) were the optimal patterns for RF. The patterns including adduction and external rotation components (D1F, D2E) for VMO and BF, and abduction and internal rotation patterns (D1E, D2F) for VL were the optimum. Although our results on RF and VMO confirmed the description of Voss et al., BF showed higher muscle activities in patterns with extension components. As reported by Basmajian et al. ${ }^{14)}$ and Adler et al. ${ }^{15)}$, BF is active synergistically as the hip extensor muscle, therefore, BF may be strongly affected by patterns with extension components (D1E, D2E). We could not confirm that the patterns with abduction and internal rotation components (D1E, D2F) were optimal for VL. This was consistent with the study by Arsenault et al. ${ }^{13)}$.

The muscle activity observed in the present study, should be considered when selecting an optimal PNF pattern for a given muscle. Furthermore, detailed analysis of muscle activities in different PNF patterns may be especially important for selecting an optimal pattern.

\section{REFERENCES}

1) Voss DE, et al: Proprioceptive Neuromuscular Facilitation. Patterns and Techniques. 3rd ed, New York: Harper \& Row Inc, 1985.

2) Nakajima H, et al: Sports PNF Manual Therapy and Training. Tokyo: Nankodo, 1992 (in Japanese).

3) Yanagisawa K: Proof of PNF effects. Rigaku ryohogaku 18(3): 354-358, 1991 (in Japanese).

4) Svendsen DA, et al: Facilitation of the isometric 
maximum voluntary contraction with traction. A test of PNF predictions. Am J Phys Med 62: 27-37, 1983.

5) Yanagisawa K, et al: Influence of shoulder PNF starting positions on behavioral aroused. Rigaku ryohogaku 18(2): 143-144, 1991 (in Japanese).

6) Fujita M, et al: The effect of PNF position of the upper extremity on rapid knee extension. Tohoku J exp Med 150: 31-35, 1986.

7) Marjorie AM, et al: Depression of Hoffmann reflexes following voluntary contraction and implications for proprioceptive neuromuscular facilitation therapy. Phys Ther 71(4): 321-329,1991.

8) Nelson AG, et al: Proprioceptive neuromuscular facilitation versus weight training for enhancement of muscular strength and athletic performance. J Orthop Sports Phys Ther 8: 250-253,1986.

9) Osternig LR, et al: Differential responses to proprioceptive neuromuscular facilitation (PNF) stretch techniques. Med Sci Sports Exerc 22(1): 106111,1990 .

10) Wang R-Y: Effect of proprioceptive neuromuscular facilitation on the gait of patients with hemiplegia of long and short duration. Phys Ther 74(12): 11081115, 1994.

11) Markos PD: Ipsilateral and contralateral effects of proprioceptive neuromuscular facilitation techniques on hip motion and electromyographic activity. Phys Ther 59(11): 1366-1373,1979.

12) Sullivan PE, et al: Electromyographic activity of shoulder muscles during unilateral upper extremity proprioceptive neuromuscular facilitation patterns. Phys Ther 60(3): 283-288,1980.

13) Arsenault $A B$, et al: An electromyographic investigation of the individual recruitment of the quadriceps muscles during isometric contraction of the knee extensors in different patterns of movement. Physio Can 26(5): 253-261,1974.

14) Basmajian JV, et al: Muscles Alive. 5th ed, Baltimore: Williama \& Wilkins Co, 1985.

15) Adler SS, et al: PNF in Practice. An Illustrated Guide. 1st ed, New York: Springer-Verlag, 1993. 